\title{
Does a color difference between parts impair the perception of a whole? A similarity between simultanagnosia patients and healthy observers
}

\author{
Liat Goldfarb • Anne Treisman
}

Published online: 6 July 2011

(C) Psychonomic Society, Inc. 2011

\begin{abstract}
In three experiments, participants decided whether a Star of David shape was present among distractors. Although the participants were instructed to ignore the colors in the display, detection was slower when each triangle of the Star of David was printed in a different color than when the Star of David was printed in a uniform color or when each triangle was in two colors. Extending the object file theory, we suggest that when the parts of an object are distinguished by a color difference and are perceived as separate objects, the perception of the whole object, which is composed of these same parts, is damaged. One interpretation within object file theory is that when the visual system represents the location of a complex object as occupied by identity tags for its different parts, it cannot also link the same location to the identity of the complex object. A new object file must then be created.
\end{abstract}

Keywords Object file · Perception · Visual search .

Simultanagnosia

Patients with simultanagnosia have difficulty in identifying two stimuli presented simultaneously. In 1959, Luria (1959) asked a simultanagnosia patient who had Balint's syndrome to identify different objects. He found that when a Star of David was printed in a uniform color, the patient identified it correctly. However, when each triangle of the Star of David was printed in a different color, the patient could see only one triangle at a time. How does this finding compare

L. Goldfarb $(\bowtie) \cdot$ A. Treisman

Department of Psychology, Center for the Study of Brain, Mind, and Behavior, Princeton University,

Green Hall,

Princeton, NJ 08544, USA

e-mail: lgoldfar@princeton.edu with the way the healthy brain operates? Although the environment is rich in mixed-color objects, are they normally harder to identify?

The difficulty simultanagnosic patients have can be defined as an inability to see two objects at once. But what counts as an object? An object can be formed in many ways of which grouping may be one (e.g., Feldman, 1999; Scholl, 2001). Grouping by similarity is a common phenomenon in which similar elements are grouped together intentionally or automatically to form a single object. In particular, color similarity can be a basis for grouping (see Rock \& Palmer, 1990, for a review). Gilchrist, Humphreys, Riddoch, and Neumann (1997) presented items with noncollinear edges and found that grouping occurred quickly and efficiently when they were printed in the same contrast but did less so when they were printed in opposite contrast (i.e., one brighter and one darker than the background). Similarly, Beaudot and Mullen (2001) found that grouping Gabor elements together is easier when the stimuli are achromatic than when they are in different colors. Grouping elements by color similarity may form a single object even before the specific elements that construct it have been individuated (Goldfarb \& Treisman, in press).

It has been documented that when an object is composed of parts with opposite contrasts or with different colors, it can slow perception of the object (e.g., Beaudot \& Mullen, 2001; Gilchrist et al. 1997). But how exactly might difficulty in grouping interfere with perception of the whole? One possibility is that any color difference in a shape may make it harder to perceive, simply because it breaks up the grouping of the lines or borders. A more specific difficulty may arise when the color difference segregates parts of the object that are not its natural parts. Figure 1 gives an example. Here, grouping by color creates 


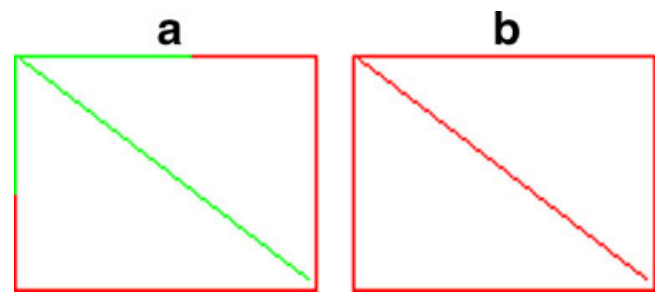

Fig. 1 Example of unnatural segregation of parts introduced by a color difference within an object

an arrow and additional lines. We can assume that "an arrow with additional lines" is not the natural way the uniform color shape in Fig. 1b is perceived.

According to some accounts (e.g., Sutherland, 1968), the perceptual representation of a shape consists of a structural description of its parts and their spatial relations. Some possible decompositions of a complex shape are more natural than others. For example, the Star of David could be perceived as a hexagon and six small triangles, but this is less natural than the perception of two superimposed triangles. Kosslyn (1980, as cited in Kirby \& Kosslyn, 1990) showed that it is easier to visualize the image of the star of David when participants are instructed to memorize it as being composed of two triangles than as a hexagon with six small triangles. Similarly, Reed (1974) showed that recognition of a part after participants are shown a whole shape is faster and more likely for natural than for unnatural parts. Both provide evidence that the Star of David is naturally described as two superimposed triangles.

If the initial representation of a complex object is normally built up from its parts, making the natural parts salient by coloring each triangle in different color might actually help rather than hinder perception of the whole. There is no documentation on the way within-color grouping affects perception in normal participants when it creates congruent parts. Luria's (1959) patient shows that in simultanagnosia, making a part salient by color contrast preempts perception of the whole, but this could be because they are unable to see two objects at a time. Would normal, healthy observers also show this difficulty with the Star of David segregated in two colors, or might the color segregation of the parts actually facilitate the natural structural description and thereby speed its perception relative to a uniform, colored Star of David?

We asked participants to detect a Star of David shape among distractors. The Star of David was printed either in a uniform color or in two colors, so that each triangle was printed in a different color. By comparing the latency to detect the target in the bicolored and in the monochromatic presentation, we could test whether perception was impaired when the parts were more likely to be perceived first.

Note that in the present task, participants were instructed to search for the Star of David shape while ignoring the color dimension. If features are represented in their own specialized feature "maps" independently from other features (e.g., Treisman, 2006; Treisman \& Gelade, 1980; Treisman \& Sato, 1990), the search for the shape could theoretically be performed independently from the color dimension as the task demands. If a difference between the experimental conditions is found, it will also suggest that the search is not performed at the level of the feature maps. The result would imply that differently colored parts within the shape are automatically detected and may interfere with or facilitate the identification of the whole.

\section{Experiment 1}

In Experiment 1, participants were asked to search for a Star of David in a display that contained nine objects. The target - the Star of David — was printed either in uniform color (red or green) or in mixed colors so that each triangle was printed in a different color (red and green). The participants were instructed to ignore the color dimension. Reaction times (RTs) were then compared between the detection of the Star of David in the mixed-color condition and in the uniform-color condition.

\section{Method}

Participants Six undergraduate students from Princeton University, with normal or corrected-to-normal vision, participated in the experiment in partial fulfillment of course requirements.

Stimuli The stimuli were presented in a rectangular display, $14.9^{\circ} \mathrm{W} \times 11.1^{\circ} \mathrm{H}$, composed of three rows and three columns in which nine colored items appeared. The size of each item was approximately $3.6^{\circ} \mathrm{W} \times 2.7^{\circ} \mathrm{H}$. The display appeared in the center of a black screen. The items that appeared in the display were either a Star of David or distractors consisting of triangles facing up, triangles facing down, and a Star of David look-alike shape that was similar to the Star of David but had an additional tilted line (see Fig. 2). The two possible colors were red and green. One triangle in each of the composite items was printed in one color, and the rest of the shape was printed either in the same or in a different color, approximately matched in their contrast. When the Star of David was present, in half of the trials, it was printed in a uniform color, and in the rest, its two component triangles appeared in different colors. The specific targets and distractors that appeared in each location were selected randomly for each trial from a list in which each appeared in each location an equal number of times across the whole experiment. 
Fig. 2 Examples of three types of displays in Experiment 1. In the left display, the target appears in uniform color; in the middle display, the target appears in mixed colors, and in the right display, the target is absent
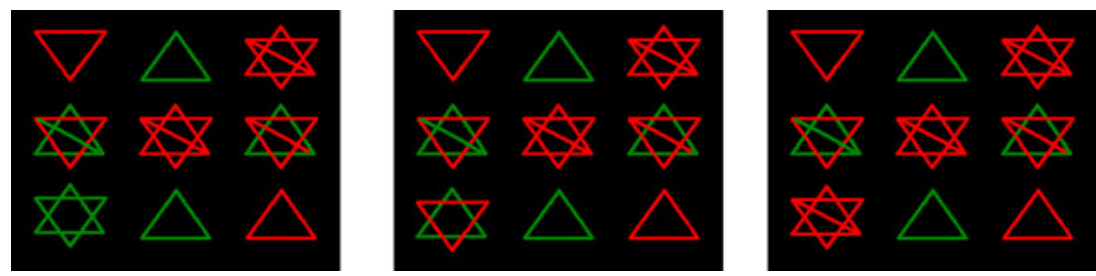

Procedure Stimulus presentations and data collection were controlled by a Dell computer with an Intel Xeon central processor. Stimuli were presented on a Dell 19-in. monitor. A keyboard was placed on a table between the participants and the monitor. Participants were run individually. They sat approximately $65 \mathrm{~cm}$ from the computer screen. Participants were instructed to detect whether a Star of David shape appeared in the display while ignoring the colors the shapes were printed in (red, green, or mixed colors). The participants were asked to respond as fast as possible, but to avoid mistakes. In the instruction phase, participants were introduced to Fig. 2 so that they would become familiar with the variation of the targets and distractors.

Each trial started with a fixation point, a white plus sign, at the center of a black screen for $1,000 \mathrm{~ms}$, followed by the nine-item display. If the Star of David appeared in the display, participants pressed the "p" key, and if it did not appear, they pressed the " $q$ " key. The items disappeared when the participants responded or after $5,000 \mathrm{~ms}$, and then the next trial began. The computer registered the participant's responses as well as the RT in milliseconds from the item's onset to the participant's response.

Before beginning the experimental block, participants were given four trials of practice, and then performed a block of 108 experimental trials. From the 108 experimental trials, 36 did not display the Star of David, 36 displayed a uniform color Star of David, and 36 displayed a mixedcolor Star of David. The duration of the experiment was approximately $5 \mathrm{~min}$.

\section{Results and discussion}

The overall mean error rate was $2.9 \%$ for uniform color targets and $5.3 \%$ for mixed-color targets. The false-alarm rate on target-absent trials was 3.4\%. For the correct trials, mean RTs were calculated for each participant for the uniform color target and for the mixed-color target conditions. A paired-sample $t$ test was applied to these data. Uniform color targets were detected significantly faster $(1,439 \mathrm{~ms})$ than were mixed color targets $(1,601 \mathrm{~ms}) t(5)=3.39 p<.02$. In addition, since in the present experiment we used only six participants, we also inspected the RTs of individual participants, which suggested that for every individual, the uniform condition was perceived faster than the mixed condition. To sum up, the analysis suggests that it is easier to detect a shape when it is printed in a uniform color than in mixed colors.

\section{Experiment 2}

Experiment 2 was designed to generalize the findings of Experiment 1. In this experiment, we verified that the effect is not restricted to the colors red and green, to the specific proportions of the target appearance $(66.6 \%$ of the trials in Experiment 1), and to situations in which the color of the target was the same as the color of the distractors in the same display.

\section{Method}

Experiment 2 was similar to Experiment 1, except for the following changes. Twelve undergraduate students participated in the experiment. In the present experiment, the stimuli were printed using more varied colors. In addition, to the red and green colors that were used in Experiment 1, we added the following colors: gray, orange, pink, purple, white, azure, brown, and yellow. When mixed items appeared (the Star of David targets or the Star of David look-alike distractors), they appeared in the following combinations: red-green (as in Experiment 1), purple-orange, and white-pink. The targets were equally often mixed colors from the three pairs (red/ green, white/pink and orange/purple) and uniform colors chosen from the same set of six colors. In addition, no target color also appeared in any of the distractors in the same display (see Fig. 3). Except for this constraint, the distractor colors were selected randomly for each trial. In the instruction phase, participants were shown Fig. 3 to familiarize them with the variation of the targets and distractors. The experimental block had 216 experimental trials. In half of them, the target was present. The duration of the experiment was approximately $9 \mathrm{~min}$.

\section{Results and discussion}

The overall percent of missed targets was $4.6 \%$ for uniformcolor targets and $7.8 \%$ for mixed-color targets. The falsealarm rate on target-absent trials was $4.6 \%$. For the correct trials, mean RTs were calculated for each participant for the uniform-color target and for the mixed-color target conditions. A paired-sample $t$ test was applied to these data. Uniform 
Fig. 3 Examples of three types of displays in Experiment 2. In the left display, the target appears in uniform color; in the middle display, the target appears in mixed colors, and in the right display, the target is absent
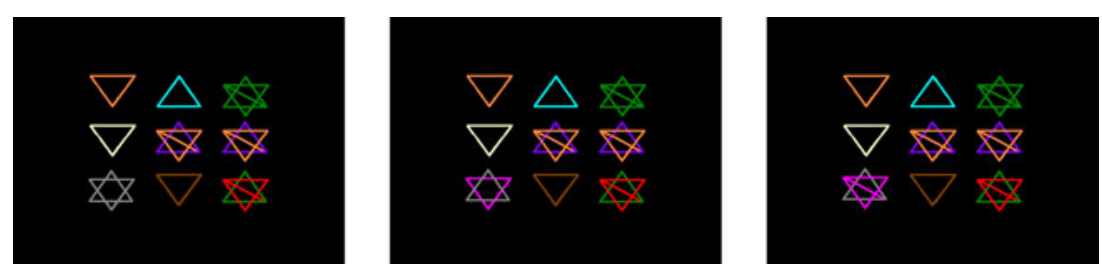

color targets were detected significantly faster $(1,195 \mathrm{~ms})$ than mixed color targets $(1,295 \mathrm{~ms}) t(11)=3.07 p<.01$.

In addition, we examined whether the effect was driven by a single combination of colors. To address this issue, a two-way ANOVA was applied to the RT data with target color or pair and uniform/mixed condition as withinsubjects factors. This interaction failed to reach significance, $F_{-}<1$. This suggests that the effect is not limited to a single combination of colors.

To sum up, the present results suggest again that it is easier to detect a shape when it is printed in uniform color than in mixed colors. This effect can be observed both when the distractors are printed in the same color as the target and also when they are printed in a variety of different colors.

\section{Experiment 3}

The previous experiments demonstrated that when perceptual parts of the Star of David (the two triangles) are printed in different colors, the perception of the star is damaged. The present experiment was designed to test how far the difficulty is due to mixing colors within an object and how far it results from the segregation of the object into its natural parts. We compared the detection time of a Star of David in which the two triangle parts were printed in different uniform colors to a Star of David in which parts within each triangle were printed in different colors. When the contours of a shape are broken up, it may be harder to perceive the shape. Hence, we created displays in which all of the contours were discontinuous. Within these displays, the Star of David target could be printed either in uniform color or with each triangle in a different uniform color (e.g., one triangle was green and the other one was red), or with the contours of the whole shape broken into different colored segments, (see Fig. 4 for the different displays). RTs for the different conditions were measured and then compared.

\section{Method}

Stimulus presentations and data collection were controlled by a Dell laptop computer: Latitude D830 with an Intel central processor and 15-in. monitor. Six volunteers, with normal or corrected-to-normal vision, participated in the experiment. The method of Experiment 3 was the same as that of Experiment 1, except for the following changes. The stimuli in this design were similar to the stimuli in Experiment 1, but they were composed of discontinuous lines. Each triangle's sides as well as the additional tilted line in the Star of David look-alike shape were composed of six separated line segments. Three experimental conditions were included in this experiment. In the first condition, the target Star of David appeared in a uniform color. In the second condition, one triangle of the target appeared in one color, and the other in a different color. In the third condition, each of the two triangles was printed in two different alternating colors. The first two conditions were comparable to the two conditions in Experiment 1. A comparable condition to the third condition did not appear in Experiment 1. Four types of distractors were included in each display, all consisting of broken lines. The four types were a uniform-color red, a uniform-color green, two colors with triangles segregated, and two colors in alternating contours. As in the previous experiments, the specific targets and distractors that appeared in each location were

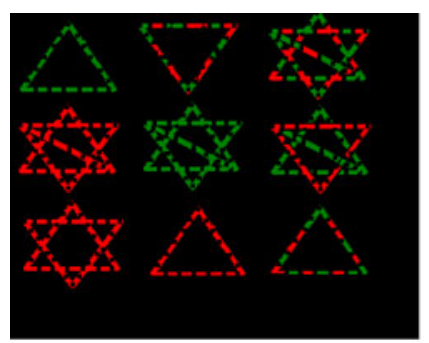

a

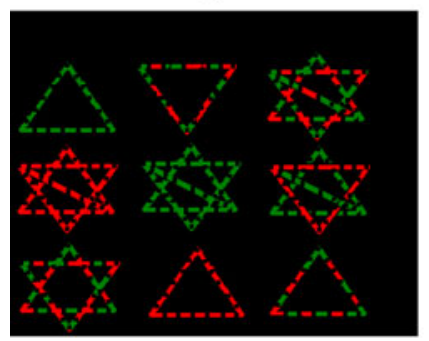

C

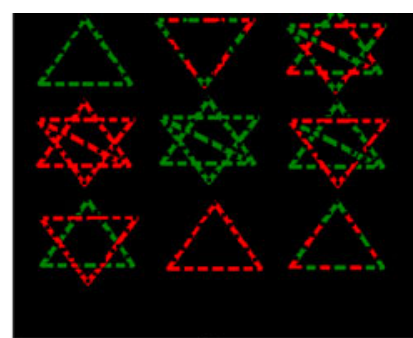

b

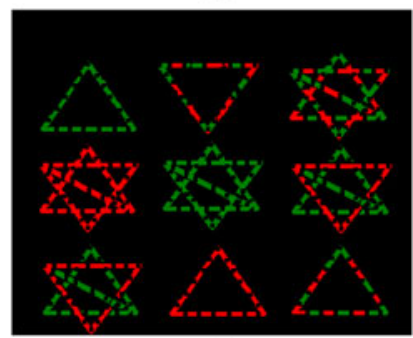

d
Fig. 4 Examples of the four types of displays in Experiment 3. a The target appears in uniform color. b The target appears in mixed color: each triangle is printed in a different color. c The target appears in mixed color: each triangle is printed in two colors. $\mathbf{d}$ The target is absent 
selected randomly for each trial from a list in which each appeared in each location an equal number of times. See Fig. 4 for examples of the different stimuli and the different conditions used in this experiment.

Participants were given four trials of practice, and then performed a block of 162 experimental trials. From the 162 experimental trials, 54 did not contain the Star of David, 36 contained a uniform-color Star of David, 36 contained a mixed-color Star of David in which each triangle was printed in different colors, and 36 contained a mixed-color Star of David in which each triangle was printed in two different colors. The duration of the experiment was approximately $7 \mathrm{~min}$.

\section{Results and discussion}

The overall percent of missed targets was $1.9 \%$ for uniform-color targets, $3.4 \%$ for the mixed-color targets in which each triangle was printed in a different color, and $2.7 \%$ for mixed-color targets in which each triangle was printed in two colors. The false-alarm rate on target-absent trials was $1.8 \%$. For the correct trials, mean RTs were calculated for each participant for each condition. A oneway ANOVA was applied to these data and revealed a significant effect, $\underline{F}(2,10)=10.63, M S E=7,919, p<.01)$. Further analysis revealed that as in the previous experiment, uniform color targets were detected significantly faster $(1,666 \mathrm{~ms})$ than mixed-color targets in which each triangle was printed in a different color $(1,890 \mathrm{~ms}), \underline{F}(1,5)=23.10$, $M S E=6,506, p<.01$. In addition, mixed-color targets in which each triangle was printed in two colors were detected significantly faster $(1,711 \mathrm{~ms})$ than mixed-color targets in which each triangle was printed in a different color, $F(1,5)=$ $17.81, M S E=5,399, p<.01$.

In addition, we looked to see whether there was a decrement due to mixing the colors within lines relative to the uniform color shape, and found that this difference (45 ms) was not significant. Overall, the results suggest that mixing colors is more detrimental when it makes the parts more salient. The effect of mixing colors without segregating them in separate parts is much smaller.

\section{General discussion}

Luria (1959) demonstrated that a Balint's patient with simultanagnosia was unable to see a Star of David shape when each component triangle of the Star of David was printed in a different color. The patient could identify the overall shape correctly only when the shape was printed in a uniform color. Following Luria's study, we suggest that objects with parts in different colors may also be harder to identify for normal participants.
The present study was composed of three experiments in which participants were asked to decide whether, in a series of displays, a Star of David shape was presented among distractors. We found that when the Star of David was printed in a uniform color, it was detected faster than when each triangle within the Star of David was printed in a different color, even when participants were instructed to ignore the color dimension. This effect was found both when one or both triangles of the target shape were printed in the same color as the distractors (Experiment 1) and when the target never had the same colors as the distractors in the same display (Experiment 2). The observed difficulty must have a component that is due to the fact that the color segregated the parts, making each one a distinct object, since a difficulty in detection was also found when the target with segregated color parts was compared with other mixed-color targets (Experiment 3).

Overall the results suggest that when different colors within an object create distinct parts within the object, it is harder to perceive the object as a whole. The findings suggest that segregation can damage perception even when it results in creating elements that are congruent with the naturally perceived elements. The results of Experiment 3 imply that this result is not only due to the mixed colors of the objects, but that it has something to do with the parts being grouped into separate distinct objects.

These findings can be interpreted within the object file theory. According to the theory (Kahneman, Treisman, \& Gibbs, 1992), in order to identify an object, its different features are bound together via their location. When different objects occupy the same location in immediately successive displays, their perception is damaged relative to when the second object can be seen as a recurrence of the first (Kahneman et al., 1992). When a new object file must be created, some additional extra time is required.

When an object file is formed, its contents can be matched to a type in a recognition network, which is a stored representation of a category (such as the shape "O"). They are also bound to the location that they currently occupy. Object files can represent separate objects with identical contents, distinguished only by their current locations, but they cannot simultaneously represent different objects with different contents, within the same location. Examples supporting this generalization are the various ambiguous figures such as the Rubin vase-faces (Rubin, 1915) and Boring's (1930) wifemother-in-law drawing for which the perceived interpretation alternates over time. Overlapping objects in different colors can be seen in a shared general location (e.g., Tipper, 1985), but probably also only by alternating between them.

Within the object file framework, consider again the case of Luria's (1959) Star of David. When the two triangles are 
printed in the same color, one object is formed by grouping, and the identity tag "Star of David" can be attached to its location. When each triangle of the Star of David is printed in a different color, two shapes are formed. Then, a triangle tag might be attached to each one of the shapes in their specific locations. In this case, since the locations are occupied by triangle identity tags, it is not clear that the same locations can also be simultaneously linked to the "Star of David" tag. If two identity tags cannot be attached to the same location at the same time, then a new object file tagging procedure might be required. This could result in difficulties in processing shapes whose parts are made salient, for example, by a color difference.

It has been suggested that neurologically, features that belong to the same object are bound together by their synchrony of firing (e.g., Engel \& Singer, 2001; Von der Malsburg, 1999;). Although the neural synchrony hypothesis has proved controversial (e.g., Lamme \& Spekreijse, 1998; Shadlen \& Movshon, 1999), support for the hypothesis has also been documented in several experiments, both on human and on animal participants (for a review of the evidence, see Engel \& Singer, 2001; Raffone \& Wolters, 2001). Specifically, it was suggested that this synchrony could be the way the object file theory is neurologically instantiated (e.g., Hommel \& Colzato, 2009; Vogel, Woodman, \& Luck, 2001). This could explain as follows the difficulty our participants experienced in identifying the Star of David that was composed of two colored triangles: Binding the same location with both the identity of two triangles and the identity of a Star of David would require the same neurons to fire in two different synchronies at once.

Note that healthy participants, unlike the simultanagnosic patients, do finally manage to perceive both the parts of the object and the object as a whole. Since simultanagnosia results from bilateral lesions to the parieto-occipital junction (e.g., Riddoch et al., 2010; Rizzo \& Vecera, 2002), this suggests that the area of the parieto-occipital junction in healthy participants might play an important role in the ability to switch the parsing of the shape into parts versus a whole.

Author Note This research was supported by fellowships to L.G. from the Israel Science Foundation (Bikura), the Rothschild Foundation, and the Weizmann Institute of Science-Advancing women in science, and by Grants 2004 2RO1 MH 058383-04A1 and EY016975 from NIH to A.T.

\section{References}

Beaudot, W. H. A., \& Mullen, K. T. (2001). Processing time of contour integration: Therole of color, contrast and curvature. Perception, $30,833-853$.
Boring, E. G. (1930). A new ambiguous figure. The American Journal of Psychology, 42, 444-445.

Engel, A. K., \& Singer, W. (2001). Temporal binding and the neural correlates of sensory awareness. Trends in Cognitive Sciences, 5, $16-25$.

Feldman, J. (1999). The role of objects in perceptual grouping. Acta Psychologica, 102, 137-163.

Gilchrist, I. D., Humphreys, G. W., Riddoch, M. J., \& Neumann, H. (1997). Luminance and edge information in grouping: A study using visual search. Journal of Experimental Psychology. Human Perception and Performance, 23, 464-480.

Goldfarb, L., \& Treisman, A. (in press). Repetition blindness: The survival of the grouped. Psychonomic Bulletin \& Review.

Hommel, B., \& Colzato, L. S. (2009). When an object is more than a binding of its features: Evidence for two mechanisms of visual feature integration. Visual Cognition, 17, 120-140.

Kahneman, D., Treisman, A., \& Gibbs, B. (1992). The reviewing of object files: Object-specific integration of information. Cognitive Psychology, 24, 175-219.

Kirby, K. N., \& Kosslyn, S. M. (1990). Thinking visually. Mind and Language, 5, 324-341.

Lamme, V. A., \& Spekreijse, H. (1998). Neuronal synchrony does not represent texture segregation. Nature, 396, 362-366.

Luria, A. R. (1959). Disorders of "simultaneous perception" in a case of bilateral occipito-parietal brain injury. Brain, 83, 437-449.

Raffone, A., \& Wolters, G. (2001). A cortical mechanism for binding in visual working memory. Journal of Cognitive Neuroscience, 13, 766-785.

Reed, S. K. (1974). Structural descriptions and the limitations of visual images. Memory \& Cognition, 2, 319-336.

Riddoch, M. J., Chechlacz, M., Mevorach, C., Mavritsaki, E., Allen, H., \& Humphreys, G. W. (2010). The neural mechanisms of visual selection: the view from neuropsychology. Annals of the New York Academy of Sciences, 1191, 156-181.

Rizzo, M., \& Vecera, S. P. (2002). Psychoanatomical substrates of Balint's syndrome. Journal of Neurology, Neurosurgery, and Psychiatry, 72, 162-178.

Rock, I., \& Palmer, S. E. (1990). The legacy of Gestalt psychology. Scientific American, 262, 84-90.

Rubin, E. (1915). Visuell Wahrgenommene Figuren. Denmark: Gyldenalske Boghandel.

Shadlen, M. N., \& Movshon, J. A. (1999). Synchrony unbound: A critical evaluation of the binding hypothesis. Neuron, 24, 67-77.

Scholl, B. J. (2001). Objects and attention: The state of the art. Cognition, 80, 1-46.

Sutherland, N. S. (1968). Outlines of a theory of visual pattern recognition in animals and man. Proceedings of the Royal Society, 171, 297-317.

Tipper, S. P. (1985). The negative priming effect: Inhibitory priming by ignored objects. Quarterly Journal of Experimental Psychology, 37, 571-590.

Treisman, A. (2006). How the deployment of attention determines what we see. Visual Cognition, 14, 411-443.

Treisman, A., \& Gelade, G. (1980). A feature integration theory of attention. Cognitive Psychology, 12, 97-136.

Treisman, A., \& Sato, S. (1990). Conjunction search revisited. Journal of Experimental Psychology. Human Perception and Performance, 16, 459-478.

Vogel, E. K., Woodman, G. F., \& Luck, S. J. (2001). Storage of features, conjunctions, and objects in visual working memory. Journal of Experimental Psychology: Human Perception and Performance, 27, 92-114.

Von der Malsburg, C. (1999). The what and why of binding: The modeler's perspective. Neuron, 24, 95-104. 